\title{
Anti-A $\beta$ antibody treatment promotes the rapid recovery of amyloid-associated neuritic dystrophy in PDAPP transgenic mice
}

\author{
Robert P. Brendza, ${ }^{1,2}$ Brian J. Bacskai, ${ }^{3}$ John R. Cirrito, ${ }^{1,2}$ Kelly A. Simmons, ${ }^{1,2}$ Jesse M. Skoch, ${ }^{3}$ \\ William E. Klunk, ${ }^{4}$ Chester A. Mathis, ${ }^{4}$ Kelly R. Bales, ${ }^{5}$ Steven M. Paul, ${ }^{5}$ Bradley T. Hyman, ${ }^{3}$ \\ and David M. Holtzman'1,2,6

\begin{abstract}
${ }^{1}$ Department of Neurology and ${ }^{2}$ Hope Center for Neurological Disorders, Washington University School of Medicine, St. Louis, Missouri, USA ${ }^{3}$ Department of Neurology, Massachusetts General Hospital, Charlestown, Massachusetts, USA. ${ }^{4}$ Departments of Psychiatry and Radiology, University of Pittsburgh, Pittsburgh, Pennsylvania, USA. ${ }^{5}$ Neuroscience Discovery Research, Eli Lilly and Co., Indianapolis, Indiana, USA. ${ }^{6}$ Department of Molecular Biology and Pharmacology, Washington University School of Medicine, St. Louis, Missouri, USA.
\end{abstract}

\begin{abstract}
Neuritic plaques are a defining feature of Alzheimer disease (AD) pathology. These structures are composed of extracellular accumulations of amyloid- $\beta$ peptide $(A \beta)$ and other plaque-associated proteins, surrounded by large, swollen axons and dendrites (dystrophic neurites) and activated glia. Dystrophic neurites are thought to disrupt neuronal function, but whether this damage is static, dynamic, or reversible is unknown. To address this, we monitored neuritic plaques in the brains of living PDAPP;Thy-1:YFP transgenic mice, a model that develops AD-like pathology and also stably expresses yellow fluorescent protein (YFP) in a subset of neurons in the brain. Using multiphoton microscopy, we observed and monitored amyloid through cranial windows in PDAPP;Thy-1:YFP double-transgenic mice using the in vivo amyloid-imaging fluorophore methoxy-X04, and individual YFP-labeled dystrophic neurites by their inherent fluorescence. In vivo studies using this system suggest that amyloid-associated dystrophic neurites are relatively stable structures in PDAPP;Thy-1:YFP transgenic mice over several days. However, a significant reduction in the number and size of dystrophic neurites was seen 3 days after $A \beta$ deposits were cleared by anti- $A \beta$ antibody treatment. This analysis suggests that ongoing axonal and dendritic damage is secondary to $A \beta$ and is, in part, rapidly reversible.
\end{abstract}

\section{Introduction}

Alzheimer disease (AD) is a neurodegenerative disorder that results in memory deficits, changes in personality, and cognitive decline. It is the leading cause of dementia in the US, affecting approximately $10 \%$ of those over 65 and $50 \%$ of those over 85 years of age. One of the invariant pathological hallmarks of $\mathrm{AD}$ is the presence of neuritic plaques in areas of the brain responsible for memory and cognition. Neuritic plaques consist predominantly of extracellular fibrils of amyloid- $\beta$ peptide $(A \beta)$ and are closely associated with dystrophic neurites, activated microglia, and reactive astrocytes (1-3). The actual mechanisms that contribute to the pathogenesis of $\mathrm{AD}$ are not known; however, compelling genetic and biochemical evidence suggests that accumulation of amyloid$\beta$ protein plays a central role. Thus, preventing or reversing the formation of amyloid may be a viable treatment.

The dystrophic neurites that surround amyloid deposits are markedly swollen, distorted axons and dendrites. In AD, the number of dystrophic neurites has been shown to correlate with the clinical severity of dementia (4), and neuronal dystrophy is associated with synaptic loss in cortical cultures exposed to fibrillar $\mathrm{A} \beta$ (5). Studies in human $\mathrm{AD}$ and in transgenic models suggest that alterations in dendritic curvature and morphology, including neuritic dystrophy, that are associated with deposits of fibrillar $\mathrm{A} \beta$

Nonstandard abbreviations used: $A \beta$, amyloid- $\beta$ peptide; $A D$, Alzheimer disease; CAA, cerebral amyloid angiopathy; YFP, yellow fluorescent protein.

Conflict of interest: The authors have declared that no conflict of interest exists.

Citation for this article: J. Clin. Invest. 115:428-433 (2005)

doi:10.1172/JCI200523269. are likely to profoundly effect neural network function, as ascertained by computer modeling $(6,7)$. Though neuritic dystrophy is thought to contribute to cognitive impairment by disrupting neuronal function, many physiological characteristics of dystrophic neurites in vivo remain largely unknown.

Various studies have shown that different anti-A $\beta$ immunotherapies can reduce the amount of brain $\mathrm{A} \beta$ deposits in transgenic mouse models of AD (8-19). It is not known, however, whether removal of $A \beta$ would reverse neuritic dystrophy and, if so, how rapidly this would occur. Insight into the stability of amyloid plaques, diffuse $\mathrm{A} \beta$ deposits, and cerebral amyloid angiopathy (CAA) in $\mathrm{AD}$ transgenic mouse models has been obtained by in vivo brain imaging using 2-photon microscopy (10, 11, 20-22). When monitored over a 5-month period using this technique, dense-cored amyloid plaques in the brains of living APP transgenic mice were seen to develop very rapidly and for the most part to remain stable in size and shape, while a small population of plaques appeared to undergo periods of dynamic growth and shrinkage (21). It was further shown that topical application of antibodies specific for the $A \beta$ protein to the brains of APP transgenic mice could promote clearance of diffuse $A \beta$ deposits and amyloid as monitored by 2-photon microscopy over a 3- to 8-day period $(10,11)$. Attempts have been made to observe neurite changes associated with amyloid in vivo using fluorescent dextrans to label neurites in transgenic mice (23). These studies showed that dense-cored plaques could alter neurite trajectories and disrupt the neuropil in a relatively large area surrounding the core, but this type of labeling approach was not conducive to high-resolution, long-term analysis of neuronal structures in vivo. 


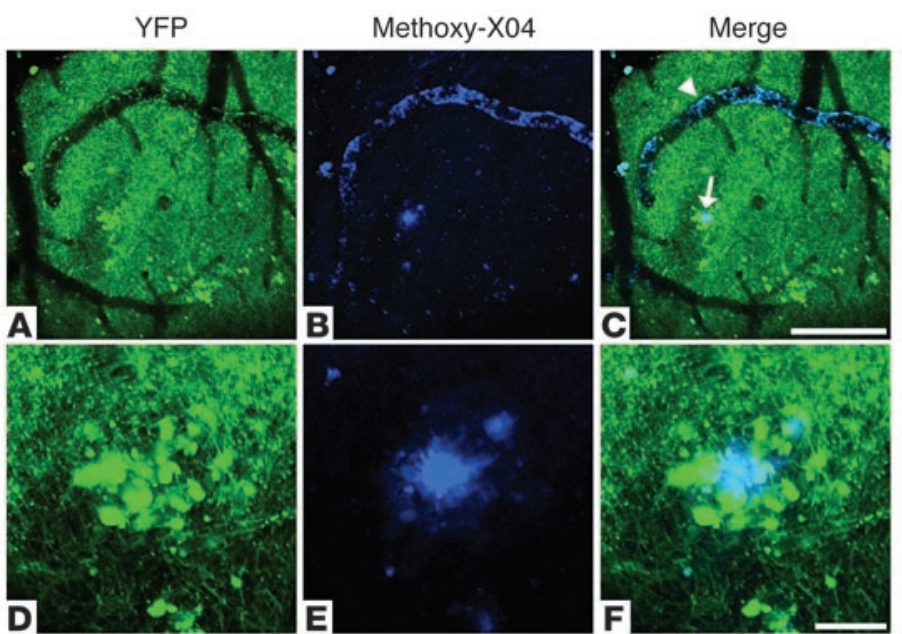

\section{Figure 1}

Neuritic plaques and CAA in a living PDAPP;Thy-1:YFP doubletransgenic mouse. Using multiphoton microscopy, we were able to observe and monitor amyloid plaques and CAA through cranial windows in PDAPP;Thy-1:YFP double-transgenic mice using the in vivo amyloid-imaging fluorophore methoxy-X04 (blue). These images were taken approximately 24 hours after an i.p. injection of methoxyX04. Amyloid-associated YFP-labeled dystrophic neurites, as well as unaffected axons, dendrites, and dendritic spines, were visualized by their inherent fluorescence (green). (A-C) A low-magnification view of an area within the cerebral cortex of a PDAPP;Thy-1: YFP double-transgenic mouse, in which prominent neuritic plaques and CAA can be seen. The arrowhead points to CAA and the arrow to a neuritic plaque in the brain parenchyma. (D-F) A higher-magnification view of a neuritic plaque found in the same area shown in A. (A and D) YFP only; (B and E) methoxy-X04-stained amyloid only; (C) merged image of $\mathbf{A}$ and $\mathbf{B}$; $(\mathbf{F})$ merged image of $\mathbf{D}$ and $\mathbf{E}$. Scale bars: A-C, $200 \mu \mathrm{m} ; \mathbf{D}-\mathbf{F}, 20 \mu \mathrm{m}$.
To study the dynamics of neurite-amyloid interactions and to investigate the properties of amyloid toxicity in vivo, we analyzed neuritic plaques in the brains of living PDAPP; Thy-1:YFP transgenic mice, a transgenic mouse model that develops AD-like pathology and also stably expresses yellow fluorescent protein (YFP) in a subset of neurons in the brain (24). In PDAPP;Thy-1:YFP doubletransgenic mice, large YFP-labeled dystrophic axons and dendrites associated with fibrillar deposits of $A \beta$ are easily visualized (24). In the present study, using multiphoton microscopy, we observed and monitored amyloid through cranial windows in PDAPP;Thy-1: $Y F P$ double-transgenic mice using the in vivo amyloid-imaging fluorophore methoxy-X04 (25), and individual YFP-labeled dystrophic neurites adjacent to amyloid were visualized over time by virtue of their inherent fluorescence. We have used this system to investigate whether amyloid-associated dystrophic neurites undergo dynamic changes over time and whether rapid clearance of $A \beta$ has an acute affect on neuritic dystrophy in living PDAPP;Thy-1: YFP double-transgenic mice.

\section{Results}

Using an in vivo imaging system, we monitored amyloid-associated dystrophic neurites in the brains of living PDAPP;Thy-1:YFP double-transgenic mice using multiphoton microscopy. In the neocortex, these mice express YFP in layer 5 neuronal cell bodies and their dendrites that extend to the cortical surface. Through cranial windows, cerebrovascular and parenchymal amyloid deposits were observed with systemically injected methoxy-X04,

\section{Figure 2}

Reimaging of a neuritic plaque in a living PDAPP;Thy-1:YFP doubletransgenic mouse. ( $\mathbf{A}$ and $\mathbf{B}) A$ single neuritic plaque imaged through a cranial window in a living PDAPP;Thy-1:YFP double-transgenic mouse with 2-photon microscopy on the initial day of surgery. (C and $\mathbf{D})$ The same plaque shown in $\mathbf{A}$ and $\mathbf{B}$, imaged 3 days after surgery. Multiple neurites of normal caliber and dystrophic neurites (arrows) were easily visualized with YFP fluorescence (green). A and $\mathbf{C}$ show both methoxyX04-labeled amyloid (blue) and YFP-labeled neurites. B and D are identical to $\mathbf{A}$ and $\mathbf{C}$, respectively, but only show the YFP signal. In the absence of any treatment, the relative number and size of amyloidassociated dystrophic neurites did not significantly change over a $72-$ hour period. Each panel is a projection of multiple focal planes that span approximately $20 \mu \mathrm{m}$. Scale bar: $20 \mu \mathrm{m}$. and YFP-labeled dystrophic neurites were visualized by their inherent fluorescence (Figure 1). Figure 1, D-F, shows a neuritic plaque with multiple large dystrophic neurites imaged in vivo on the initial day of surgery. The exact location of each neuritic plaque analyzed was mapped by virtue of crude stereotaxic coordinates and more precisely by their relative positions to cerebral blood vessels (either affected or unaffected by CAA) and other stable fiduciary markers in the vicinity of the plaque (Figure 1, A-C). This mapping system enabled us to reproducibly identify the same plaques in living PDAPP;Thy-1:YFP double-transgenic mice during imaging sessions that were separated by days to weeks (Figure 2).

Using this system, we first analyzed neuritic pathology in living double-transgenic mice in the absence of any treatment to determine whether the number or size of amyloid-associated dystrophic neurites changed dynamically over a period of 72 hours. To monitor potential changes in populations of dystrophic neurites, image stacks that encompassed neuritic plaques were generated on the initial day of surgery (day 0 ), and the same plaques were imaged again 3 days later (day 3 ). The total number and total cross-sectional area of dystrophic neurites within a plaque were
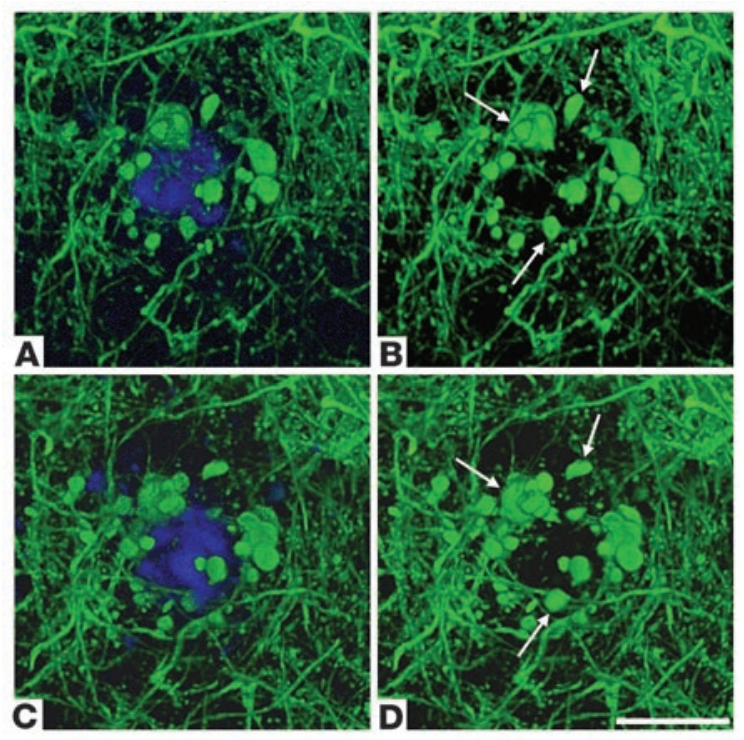


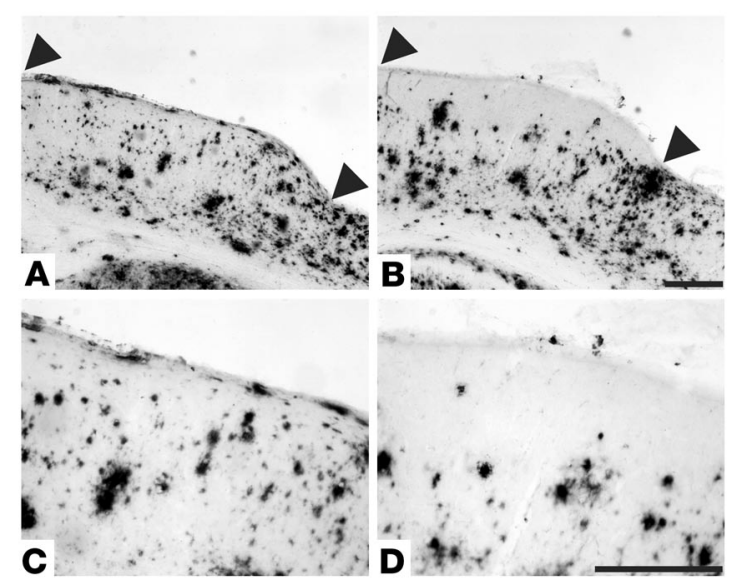

Figure 3

Clearance of $A \beta$ deposits by direct application of anti-A $\beta$ antibody to the surface of the brain. (A) $A \beta$ immunoreactivity in a coronal section from the brain of a PDAPP;YFP double-transgenic mouse that received no treatment. The mouse was fitted with a cranial window on day 0 and sacrificed 7 days later. (B) A $\beta$ immunoreactivity in a coronal section from the brain of a PDAPP;YFP double-transgenic mouse that received treatment with an $\mathrm{N}$-terminal-specific anti-A $\beta$ antibody (20 $\mu \mathrm{g}$ of 10D5). The antibody was administered directly to the surface of the brain, directly under the cranial window, during the surgical procedure on day 0 and sacrificed 7 days later. In both $\mathbf{A}$ and $\mathbf{B}$, the area of the brain exposed by the cranial window lies between the arrowheads. (C) A higher magnification of the cortical area exposed by the cranial window in the untreated brain shown in $\mathbf{A}$. (D) A higher magnification of the cortical area exposed by the cranial window in the 10D5-treated brain shown in $\mathbf{B}$. Note the region of reduced $A \beta$ immunoreactivity within 100-200 $\mu \mathrm{m}$ below the cortical surface in the treated area. Scale bars: $250 \mu \mathrm{m}$.

determined for each time point. A dystrophic neurite was defined as any enlarged YFP-labeled neurite measuring more than $2.5 \mu \mathrm{m}$ in diameter. The total dystrophic-neurite cross-sectional area for a given plaque represents the sum of the largest cross-sectional area for each individual dystrophic neurite found within the plaque. This last measure is influenced by both number and size of individual dystrophic neurites associated with a plaque. Statistical analysis of image data from 23 neuritic plaques in 10 different PDAPP;YFP mice indicates that dystrophic neurites did not undergo major changes in number or size over a 72 -hour period in the absence of any treatments (see Figure 5).

We next wanted to determine whether an anti-A $\beta$ antibody that reduces the amount of diffuse $A \beta$ and amyloid in the brains of PDAPP transgenic mice would promote the rapid recovery of neuritic dystrophy. In previous studies, $\mathrm{N}$-terminal-specific anti-A $\beta$ antibodies have been shown to decrease $A \beta$ in a region-specific manner in the brains of PDAPP transgenic mice over a 7- to 8-day period when administered to the brain surface $(10,11,16)$. Using one of these anti-A $\beta$ antibodies, 10D5, we also found a reduction in the amount of $\mathrm{A} \beta$ deposits in the brains of PDAPP;Thy-1:YFP transgenic mice in the immediate vicinity of the application of the antibody to the cortical surface after 7 days (Figure 3 ). We sought to determine whether application of 10D5 to the cortical surface had any effect on the large dystrophic neurites surrounding amyloid deposits compared with application of vehicle alone. Interestingly, in mice receiving $10 \mathrm{D} 5$, there was a rapid morphological recovery of some dystrophic neurites that could be observed over as short a period as 3 days (Figure 4). While most of the largest dystrophic neurites did not appear to change dramatically over this period, the smaller dystrophic neurites were often more shrunken, and some were no longer observable. In some instances, a neuritic swelling that had been present on a normal-appearing dendrite or axonal segment disappeared (Figure 4). Statistical analysis of 18 plaques from 7 different PDAPP;Thy-1:YFP transgenic mice demonstrated that a significant decrease in both number and total cross-sectional area of dystrophic neurites occurred after 10D5 treatment (Figure 5). Several PDAPP;Thy-1:YFP transgenic mice receiving $10 \mathrm{D} 5$ treatment were followed out to 1 week; the diminution in neuritic dystrophy persisted, and in some cases the number of dystrophic neurites appeared to decrease relative to the 3-day time point (data not shown). Based on these data, it appears that axonal and dendritic structural damage associated with amyloid deposits is not permanent and is, at least in part, reversible over a relatively short time frame. Further, the specificity of the antibody shows that $A \beta$ itself causes these reversible structural changes.

Because of the rapid change in dystrophic neurites induced by $10 \mathrm{D} 5$, we wanted to know whether application of this antibody resulted in a change in total $A \beta$ immunostaining as well as fibrillar $A \beta$. Since it was difficult to quantitate fibrillar deposits in vivo using methoxy-X04 in PDAPP;Thy-1:YFP mice, we administered $10 \mathrm{D} 5$ to an additional 4 17- to 18-month-old PDAPP mice and assessed $A \beta$ and thioflavine-S-positive staining in the cortex ipsiand contralateral to antibody application. There was a significant decrease in both $A \beta$-positive and thioflavine-S-positive load in the cortex ipsilateral to $10 \mathrm{D} 5$ application (mean \pm SEM; A $\beta$ load: treated/untreated cortex $=0.51 \pm 0.13, P=0.03$; thioflavine-S load: treated/untreated cortex $=0.62 \pm 0.107, P=0.04$ ).

\section{Discussion}

The amyloid hypothesis of AD proposes that the onset and progression of $\mathrm{AD}$ pathogenesis is primarily influenced by the accumulation of $A \beta$ in the brain (26). Attention has been given recently to the possibility of using $A \beta$-related immunotherapy to affect this accumulation (27). To test whether an anti-A $\beta$ antibody could actually influence structural neuronal damage associated with neuritic pathology, we monitored amyloid-associated dystrophic neurites in living PDAPP;Thy-1:YFP double-transgenic mice treated
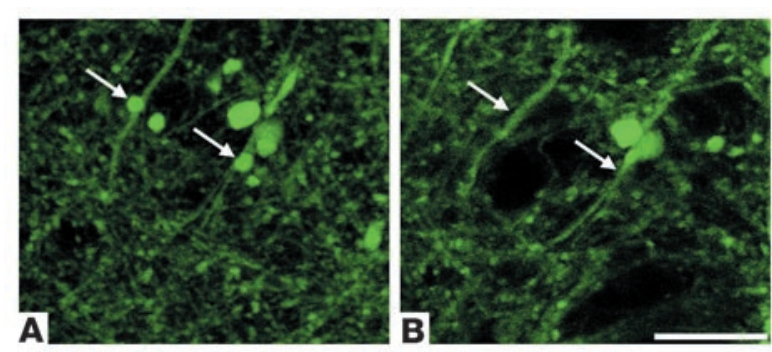

\section{Figure 4}

Morphological recovery of amyloid-associated neuritic dystrophy in a PDAPP;Thy1:YFP double-transgenic mouse after anti-A $\beta$ antibody (10D5) treatment. The 2 panels show the same population of YFPlabeled neurites, which are associated with a neuritic plaque imaged on the initial day of surgery (A) and 72 hours later (B). The antibody was administered directly to the surface of the brain during the cranialwindow surgical procedure on day 0 . The arrows in the day 0 image (A) point to 2 enlarged dystrophic neurites, which are absent in the day 3 image (B). The associated amyloid is not shown. Scale bar: $20 \mu \mathrm{m}$. 
A
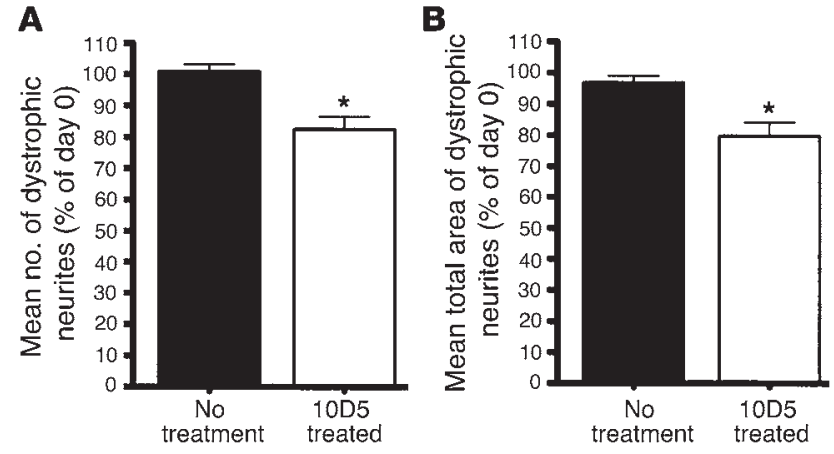

Figure 5

Repeated-measures ANOVA on data derived from 23 neuritic plaques imaged from 10 different untreated PDAPP;YFP transgenic mice and 18 plaques imaged from 7 different 10D5-treated PDAPP;Thy-1:YFP transgenic mice. All dystrophic neurites were imaged on day 0 and then reimaged on day 3 . A significant treatment $x$ day interaction for both the mean number $\left[F_{1,28}=25.092, P=0.00003\right]$ of dystrophic neurites $(\mathbf{A})$ and the mean total cross-sectional area $\left[F_{1,28}=21.037\right.$, $P=0.00009$ ] of dystrophic neurites $(B)$ was seen. In the absence of treatment, dystrophic neurites did not undergo significant changes in regard to number or cross-sectional area over a 72-hour period. However, a significant decrease in both mean number and mean cross-sectional area was seen in animals receiving 10D5 treatment. ${ }^{*} P<0.00005$ versus untreated group.

with the anti-A $\beta$ mAb 10D5. In previous studies, 10D5 treatments were shown to cause a local, rapid decrease in the amount of $A \beta$ deposits in the brains of APP transgenic mice when administered to the brain surface $(10,11,16)$. Using the combination of YFP to label neurites and methoxy-X04 (25) to label amyloid, we were able to efficiently monitor morphologically normal and dystrophic amyloid-associated neurites over time with high resolution using 2-photon microscopy in vivo.

In this study, we found that the amount of plaque-associated neuritic dystrophy did not change significantly over a 3-day period in control animals. This is consistent with observations made in live brain slices taken from PDAPP;Thy-1:YFP double-transgenic mice in which dystrophic neurites associated with neuritic plaques did not exhibit dramatic morphological changes when imaged over short time periods with fluorescence confocal microscopy (28). The apparent stability of neuritic dystrophy, in both models, probably reflects the chronic nature of this type of injury that develops over a period of days to months. Conversely, when animals received the anti-A $\beta$ antibody treatment, a rapid reversal of amyloid-associated neuritic dystrophy was seen. This result was striking in that it demonstrates that altering brain $\mathrm{A} \beta$ can facilitate an acute response to chronically injured axons and dendrites. It also suggests that the confirmed presence of uncleared $A \beta$ in the brain contributes to ongoing structural damage to neurites and is required for at least some of this damage to persist.

The exact mechanism of the effect elicited by direct application of 10D5 antibody to the brain surface is unclear, although several possibilities exist. Recent studies have demonstrated that anti-A $\beta$ antibody-induced clearance of deposited $\mathrm{A} \beta$ appears to facilitate the correction of abnormal plaque-associated neurite curvatures in PDAPP transgenic mice (16) and promotes the clearance of early tau pathology in the hippocampal regions of $3 x \mathrm{Tg}-\mathrm{AD}$ mice (17), a transgenic model that develops both amyloid plaques and neurofibrillary tangles (29). Oddo et al. (29) showed that anti-A $\beta$ antibody injections into the hippocampus resulted in decreased tau staining in the somatodendritic compartment of neurons by 5 days. While their study demonstrated that $A \beta$ clearance could modify the accumulation of cytoplasmic protein staining for tau, it did not address whether the anti-A $\beta$ antibodies could elicit a change in the enlarged, distorted axons and dendrites that surround amyloid plaques (i.e., the structural change we know as neuritic dystrophy). Our study and that of Oddo et al. complement each other in that both show that $A \beta$ clearance may enable the recovery of a specific abnormality associated with $A \beta$ deposition. The pathological changes that we were able to assess as well as the methods that we used allowed us to evaluate an important aspect of AD-like pathology that was not amenable to prior investigations. By using multiphoton microscopy and intrinsically labeled neuronal processes, we were able to detect decreases in the size of enlarged axonal and dendritic swellings in individual plaques over time. This effect may be due to clearance and/or neutralization of deposited $A \beta$. A recent study using electrophysiological measurements in vivo has shown that the insoluble $A \beta$ aggregates are associated with a disruption in neocortical synaptic function that is not seen when only soluble forms are present (30). This supports not only the idea that rapid improvement in neurite structure can come from clearing of insoluble $A \beta$ deposits but also the idea that rapid shrinkage of dystrophic neurites has the potential to rapidly improve neuronal and synaptic function. It is conceivable that some of the rapid behavioral improvements seen following passive peripheral administration of anti-A $\beta$ antibodies could occur via rapid changes in dystrophic neurites $(31,32)$. It will be important in future studies to directly assess axonal, dendritic, and synaptic function in conjunction with the improvement in structural abnormalities induced by anti-A $\beta$ antibodies.

In the time frame in which our analysis was performed, the amyloid cores of the neuritic plaques as assessed by 2-photon microscopy were still present, but it was not possible, with this technique, to quantify whether there was an antibody-induced decrease in amyloid. To address this, we treated a separate group of PDAPP mice with 10D5 and found that there was a significant decrease in both $\mathrm{A} \beta$-immunostained and thioflavine-S-positive plaques. In a separate group of PDAPP mice, we also assessed whether application of 10D5 to the cortex affected levels of PBSsoluble $A \beta$ over 3 days. We found that this treatment resulted in no significant change in PBS-soluble $A \beta_{40}$ or $A \beta_{42}$ in the treated versus the untreated cortex (data not shown). Taken together, these results suggest that antibody-induced decrease in the number and size of dystrophic neurites is due to an effect on fibrillar $A \beta$.

The observation that only a limited number of dystrophic areas seemed to recover may reflect the initial phases of an acute response to a single antibody treatment that might be enhanced with additional time or antibody doses. Alternatively, it may reflect differences in the state of the neuritic injury. For example, dystrophic neurites that are larger and more mature may not respond to $A \beta$ clearance as well as a newly developed dystrophic neurite. Thus, more of a benefit might be seen in younger animals that are just beginning to develop pathology. To our knowledge, this study provides the first direct evidence that reducing $A \beta$ in the brain can promote the rapid structural recovery of existing amyloid-associated neuritic dystrophy in vivo. These data have important implications regarding $\mathrm{A} \beta$ clearance as a therapeutic strategy for $\mathrm{AD}$. Our in vivo imaging model should prove to be a valuable tool to study the nor- 
mal progression of AD-like pathology in transgenic mice and to assess a wide range of clinically relevant treatments for $\mathrm{AD}$.

\section{Methods}

Animals. PDAPP + - Thy-1:YFP ${ }^{+-}$double-transgenic mice were generated as described previously $(24,33,34)$. All double-transgenic mice used for this analysis were 17-18 months of age. All animal procedures used in this study have been approved by the Institutional Review Board at Washington University School of Medicine.

Surgical procedures. The cranial-window surgical procedure was performed as described elsewhere with slight modifications $(23,35)$. Briefly, animals were anesthetized with isoflurane ( $4 \%$ induction, $1.5 \%$ maintenance) while being immobilized in a custom-made animal stereotaxic apparatus equipped with an anesthetic mask. The skin was resected from just posterior to the eyes to the base of the skull, and a hole, approximately 4-6 $\mathrm{mm}$ in diameter, was carved into the skull between bregma and lambda with a fine drill bit. After drilling, the resulting "bone cap" was carefully removed, and the dura was removed from the exposed brain region. The exposed brain region was irrigated, and an 8-mm cover glass was sealed to the skull with dental cement to cover the hole. Animals receiving antibody treatment had an anti-A $\beta$ mAb, 10D5 (10 $\mu$ l of a $2-\mathrm{mg} / \mathrm{ml}$ solution in $\mathrm{Ca}^{++}$- and $\mathrm{Mg}^{++}$-free PBS), applied to the exposed brain surface before placement and sealing of the cover glass $(16,23)$. Untreated animals used as controls underwent the same procedure as antibody-treated animals except that PBS without 10D5 antibody was applied to the brain surface.

In vivo brain imaging using 2-photon microscopy. Twenty-four hours before imaging, PDAPP;Thy-1:YFP transgenic mice were injected i.p. with $10 \mathrm{mg} / \mathrm{kg}$ methoxy-X04 to specifically label amyloid in the brain (25). Anesthetized mice with cranial windows were placed on the stage of a 2-photon microscope (LSM 510 META NLO system [Carl Zeiss Inc.] with a Chameleon Ti: Sapphire laser [Coherent Inc.]) while in our custom stereotaxic device. For simultaneous imaging of methoxy-X04 and YFP, an excitation wavelength of $860 \mathrm{~nm}$ was used. Fluorescence emission from amyloid-bound methoxy$\mathrm{X} 04$ and YFP was collected in the ranges of $435-485 \mathrm{~nm}$ and $525-650 \mathrm{~nm}$, respectively. Higher-resolution imaging of YFP-labeled neurites alone was done with an excitation wavelength of $920 \mathrm{~nm}$. Low-magnification images were taken to establish map positions of neuritic plaques relative to the vasculature and other fiduciary markers within the brain. Individual neuritic plaques were imaged with an Achroplan $\times 40$ infrared water immersion lens ( 0.8 numerical aperture; Carl Zeiss Inc.) for analysis. Images were acquired as $512 \times 512$ arrays of 8 -bit pixels in z-series stacks originating at the cortical surface and proceeding downward with a step size approximately equal to the excitation wavelength being used. Z-stack data were collected for each plaque analyzed on the day of the cranial-window surgery (day 0 ) and 72 hours after surgery (day 3 ).

Data analysis. Image stacks from each imaging session were processed and analyzed using both AIM (version 3.2; Carl Zeiss Inc.) and Metamorph (version 6.1; Universal Imaging Corp.) software. Three-dimensional reconstruction of neuritic plaques from $z$-series stacks was made using maximum-intensity projections. The resulting $3 \mathrm{D}$ images obtained at different time points for an individual plaque were rotated in space to obtain the same orientation for analysis. The total number and total cross-sectional area of dystrophic neurites within a plaque were determined for each time point. A dystrophic neurite was defined as any enlarged YFP-labeled neurite measuring more than $2.5 \mu \mathrm{m}$ in diameter. The total dystrophicneurite cross-sectional area for a given plaque represents the sum of the largest cross-sectional area for each individual dystrophic neurite found within the plaque. The number and size of dystrophic neurites associated with each plaque were compared for the 2 time points.

Statistical analysis. The data were analyzed using STATISTICA 6.0 for Windows (StatSoft Inc.), and levels of $P<0.05$ were set for significance. Data regarding the mean number and mean total cross-sectional area of dystrophic neurites were analyzed with repeated-measures ANOVAs that included 1 within-subjects factor (day 0 vs. day 3 ) and 1 between-subjects factor (treatment yes vs. no). $P$ values for comparisons exceeding Bonferroni corrected levels $(0.05 / 2=0.025)$ are signified in Figure 5 by asterisks.

Histological analysis. When in vivo analysis was completed on an animal, it received a $200-\mathrm{mg} / \mathrm{kg}$ i.p. injection of pentobarbital followed by a bilateral thoracotomy and perfusion of $4 \%$ paraformaldehyde in PBS through the left ventricle. Brains were dissected and postfixed in the same fixative overnight at $4^{\circ} \mathrm{C}$, and then incubated in $30 \%$ sucrose in PBS overnight. Brains were cut into $50-\mu \mathrm{m}$ sections using a freezing sliding microtome. Immunohistochemical localization of $\mathrm{A} \beta$ - and thioflavine-positive plaques was performed on sections containing treated and nontreated areas to determine the relative densities of $A \beta$ deposits and amyloid as described previously (24).

\section{Acknowledgments}

We thank R.E. Hartman for help with the statistical analysis and B. Kennedy, M. Parsadanian, M. Finn, A. Shah, and K. Hyrc for technical assistance. This work was supported by Missouri Alzheimer's Disease and Related Disorders grant 04-6401; American Health Assistance Foundation grant A2004-240 (to R.P. Brendza); NIH grants AG13956, AG20222, P01 NS32636 (to D.M. Holtzman), AG020570, EB00768 (to B.J. Bacskai), and AG08487; an Alzheimer's Association Pioneer Award (to B.T. Hyman); Eli Lilly and Co.; and a gift from the Alafi Family Foundation.

Received for publication September 7, 2004, and accepted in revised form November 30, 2004.

Address correspondence to: David M. Holtzman, Washington University, Department of Neurology, 660 South Euclid, Box 8111, St. Louis, Missouri 63110, USA. Phone: (314) 362-9872; Fax: (314) 362-2826; E-mail: Holtzman@neuro.wustl.edu.
1. Sisodia, S.S. 1999. Alzheimer's disease: perspectives for the new millennium. J. Clin. Invest. 104:1169-1170.

2. Golde, T.E., Eckman, C.B., and Younkin, S.G. 2000 Biochemical detection of Abeta isoforms: implications for pathogenesis, diagnosis, and treatment of Alzheimer's disease. Biochim. Biophys. Acta. 1502:172-187.

3. Selkoe, D.J. 2001. Alzheimer's disease: genes, proteins, and therapy. Physiol. Rev. 81:741-766.

4. McKee, A.C., Kosik, K.S., and Kowall, N.W. 1991. Neuritic pathology and dementia in Alzheimer's disease. Ann. Neurol. 30:156-165.

5. Grace, E.A., Rabiner, C.A., and Busciglio, J. 2002. Characterization of neuronal dystrophy induced by fibrillar amyloid beta: implications for Alzheimer's disease. Neuroscience. 114:265-273.

6. Knowles, R.B., et al. 1999. Plaque-induced neurite abnormalities: implications for disruption of neural networks in Alzheimer's disease. Proc. Natl. Acad. Sci. U. S. A. 96:5274-5279.

7. Le, R., et al. 2001. Plaque-induced abnormalities in neurite geometry in transgenic models of Alzheimer disease: implications for neural system disruption. J. Neuropathol. Exp. Neurol. 60:753-758.

8. Schenk, D., et al. 1999. Immunization with amyloidbeta attenuates Alzheimer-disease-like pathology in the PDAPP mouse. Nature. 400:173-177.

9. Janus, C., et al. 2000. A beta peptide immunization reduces behavioural impairment and plaques in a model of Alzheimer's disease. Nature. 408:979-982.

10. Bacskai, B.J., et al. 2001. Imaging of amyloid- beta deposits in brains of living mice permits direct observation of clearance of plaques with immunotherapy. Nat. Med. 7:369-372.

11. Bacskai, B.J., et al. 2002. Non-Fc-mediated mechanisms are involved in clearance of amyloid-beta in vivo by immunotherapy. J. Neurosci. 22:7873-7878.

12. Sigurdsson, E.M., Scholtzova, H., Mehta, P.D., Frangione, B., and Wisniewski, T. 2001. Immunization with a nontoxic/nonfibrillar amyloid-beta homologous peptide reduces Alzheimer's diseaseassociated pathology in transgenic mice. Am. J. Pathol. 159:439-447.

13. Morgan, D., et al. 2000. A beta peptide vaccination prevents memory loss in an animal model of Alzheimer's disease. Nature. 408:982-985.

14. DeMattos, R.B., et al. 2001. Peripheral anti-A beta 
antibody alters CNS and plasma A beta clearance and decreases brain A beta burden in a mouse model of Alzheimer's disease. Proc. Natl. Acad. Sci. U. S. A. 98:8850-8855.

15. Bard, F., et al. 2000. Peripherally administered antibodies against amyloid beta-peptide enter the central nervous system and reduce pathology in a mouse model of Alzheimer disease. Nat. Med. 6:916-919.

16. Lombardo, J.A., et al. 2003. Amyloid- $\beta$ antibody treatment leads to rapid normalization of plaque-induced neuritic alterations. J. Neurosci. 23:10879-10883.

17. Oddo, S., Billings, L., Kesslak, J.P., Cribbs, D.H., and LaFerla, F.M. 2004. Abeta immunotherapy leads to clearance of early, but not late, hyperphosphorylated tau aggregates via the proteasome. Neuron. 43:321-332.

18. Wilcock, D.M., et al. 2004. Passive amyloid immunotherapy clears amyloid and transiently activates microglia in a transgenic mouse model of amyloid deposition. J. Neurosci. 24:6144-6151.

19. Wilcock, D.M., et al. 2004. Microglial activation facilitates Abeta plaque removal following intracranial anti-Abeta antibody administration. Neurobiol. Dis. 15:11-20.

20. Christie, R., Kimchi, E., Kajdasz, S., Bacskai, B., and Hyman, B.T. 2001. Multiphoton microscopy and amyloid angiopathy. Amyloid. 8(Suppl. 1):48-50.

21. Christie, R.H., et al. 2001. Growth arrest of indi- vidual senile plaques in a model of Alzheimer's disease observed by in vivo multiphoton microscopy. J. Neurosci. 21:858-864.

22. Kimchi, E.Y., Kajdasz, S., Bacskai, B.J., and Hyman, B.T. 2001. Analysis of cerebral amyloid angiopathy in a transgenic mouse model of Alzheimer disease using in vivo multiphoton microscopy. J. Neuropathol. Exp. Neurol. 60:274-279.

23. D'Amore, J.D., et al. 2003. In vivo multiphoton imaging of a transgenic mouse model of Alzheimer disease reveals marked thioflavine-S-associated alterations in neurite trajectories. J. Neuropathol. Exp. Neurol. 62:137-145.

24. Brendza, R.P., et al. 2003. PDAPP; YFP double transgenic mice: a tool to study amyloid-beta associated changes in axonal, dendritic, and synaptic structures. J. Comp. Neurol. 456:375-383.

25. Klunk, W.E., et al. 2002. Imaging Abeta plaques in living transgenic mice with multiphoton microscopy and methoxy-X04, a systemically administered Congo red derivative. J. Neuropathol. Exp. Neurol. 61:797-805.

26. Hardy, J., and Selkoe, D.J. 2002. The amyloid hypothesis of Alzheimer's disease: progress and problems on the road to therapeutics. Science. 297:353-356.

27. Holtzman, D.M., Bales, K.R., Paul, S.M., and DeMattos, R.B. 2002. Abeta immunization and anti-Abeta antibodies: potential therapies for the prevention and treatment of Alzheimer's disease. Adv. Drug Deliv. Rev. 54:1603-1613.

28. Brendza, R.P., et al. 2003. Use of YFP to study amyloid- $\beta$ associated neurite alterations in live brain slices. Neurobiol. Aging. 24:1071-1077.

29. Oddo, S., et al. 2003. Triple-transgenic model of Alzheimer's disease with plaques and tangles: intracellular Abeta and synaptic dysfunction. Neuron. 39:409-421.

30. Stern, E.A., et al. 2004. Cortical synaptic integration in vivo is disrupted by amyloid-beta plaques. J. Neurosci. 24:4535-4540.

31. Dodart, J.C., et al. 2002. Immunization reverses memory deficits without reducing brain Abeta burden in Alzheimer's disease model. Nat. Neurosci. 5:452-457.

32. Kotilinek, L.A., et al. 2002. Reversible memory loss in a mouse transgenic model of Alzheimer's disease. J. Neurosci. 22:6331-6335.

33. Games, D., et al. 1995. Alzheimer-type neuropathology in transgenic mice overexpressing V717F beta-amyloid precursor protein. Nature. 373:523-527.

34. Feng, G., et al. 2000. Imaging neuronal subsets in transgenic mice expressing multiple spectral variants of GFP. Neuron. 28:41-51.

35. Chen, B.E., et al. 2000. Imaging high-resolution structure of GFP-expressing neurons in neocortex in vivo. Learn. Mem. 7:433-441. 